\title{
Automobilität und Gesellschaft
}

\section{Zur Verortung einer sozialwissenschaftlichen Mobilitätsforschung}

\section{Von Weert Canzler}

Zusammenfassung: Allen Nebenfolgen zum Trotz: Das Automobil formiert wie kein anderes technisches Artefakt den Alltag und die Residenzwahl der Mehrheit der Bevölkerung in den westlichen Gesellschaften. Zugleich erfährt das Auto eine hohe Attraktivität in den Regionen der nachholenden Modernisierung. Dabei sind die Grenzen des fossilen Automobilismus absehbar: Das konventionell betriebene (Privat-)Auto ist kein global verallgemeinerungsfähiges Produkt. Weder reichen dafür die Brennstoffe noch erlaubt es das Weltklima. Vor diesem Hintergrund wird eine sozialwissenschaftliche Verortung von Automobilität als „Prinzip der Moderne“ vorgenommen, die mit einem Rückgriff auf den strukturationstheoretischen Ansatz von Anthony Giddens die lebensweltliche Verankerung des Autos und seine routinehafte Nutzung zu erklären versucht. Darauf aufbauend wird eine Antwort auf die Frage nach Kontinuität oder Bruch des konventionellen automobilen Entwicklungspfades versucht. Am Ende werden Hypothesen zur sozialen Dynamik künftiger Automobilität entwickelt, insbesondere zu den Chancen und Barrieren einer potenziellen automobilen Transformation.

\section{Automobilität als soziologisches Problem}

Das Automobil ist ein technisch ausgereiftes und wirtschaftlich wie kulturell erfolgreiches technisches Produkt. Zugleich prägt es die Technostruktur moderner Gesellschaften wie kaum ein anderes technisches Produkt. Dabei zeichnet es sich durch eine doppelte Pfadabhängigkeit aus: Auf der einen Seite zeigt sich bei den Herstellern und den dort tätigen Entwicklungsingenieuren eine hohe technische Pfadabhängigkeit. Entsprechend dominant sind inkrementelle Innovationen, also Verbesserungen und Ergänzungen des überkommenen - und bewährten - Autokonzeptes. Auf der anderen Seite zeigt sich eine soziale Pfadabhängigkeit: Nutzerinnen und Nutzer von Autos eignen sich das Artefakt lebensweltlich an und integrieren es in ihre Residenz-, Berufs- und Alltagsplanung. Suburbane Siedlungsstrukturen und automobiler Lebensstil bedingen sich gegenseitig (siehe Bourdieu 1998; Sieverts 1998; Voy / Polster / Thomasberger 1991), sie bilden eine Symbiose mit den damit verbundenen Abhängigkeiten. John Urry hat prägnant zusammengefasst, was für die früh industrialisierten und wohlhabenden Länder des Westens gilt und was wie eine Realprognose für jene Regionen der Welt klingt, die sich im Prozess der nachholenden Modernisierung befinden: „Social life has been irreversible locked in to the mode of mobility that automobility both generates and which can so far only be dealt with through it further expansion and restructurings of time and space" (2007: 119).

Obwohl das Automobil Standortentscheidungen und Alltagsabläufe der Mehrheit der Bevölkerung in den westlichen Gesellschaften bestimmt und es zugleich eine hohe Attraktivität in den Regionen der nachholenden Modernisierung genießt, ist es bislang kaum Thema sozialwissenschaftlicher Diskurse. ${ }^{1}$ Es gibt Grund genug für eine vertiefte sozialwissenschaftliche Beschäftigung mit der Massenmotorisierung. Nicht zuletzt ist es der massive Konformitätsdruck, wie ihn Günther Burkart 1993 diagnostiziert hat, der das Automobil zum Treibsatz der

1 In jüngster Zeit hat sich mit der von Mimi Sheller und John Urry herausgegebenen Zeitschrift „,mobilities" eine sozialwissenschaftlich ausgerichtete Zeitschrift etabliert, was Sven Kesselring als Indiz für eine Konsolidierung der sozialwissenschaftlichen Mobilitätsforschung im angelsächsischen Kontext deutet (Kesselring 2012: 84). 
sozialen Integration in der industriekapitalistischen Gesellschaft macht. Seine These lautet daher: „Das Auto gehört zur Grundausstattung eines vollwertigen Gesellschaftsmitgliedes“ (1993: 224). Der Automobilismus als ein verbreitetes Syndrom bezeichnender Begriff umfasst in diesem Sinne neben der Technik selbst und der sie ermöglichenden Infrastruktur auch soziale Rollenerwartungen und eine vielfach gelebte Alltagspraxis. ${ }^{2}$

Die Massenmotorisierung in den früh industrialisierten Gesellschaften in der zweiten Hälfte des 20. Jahrhunderts war mit Nebenfolgen und nicht-intendierten Effekten verbunden, die wiederum zu politischen Regulierungen führten. Gesetzliche Auflagen für das Automobil haben seine Anpassungsfähigkeit erhöht, ständig wurden technische Optimierungen auf der Basis des Universalfahrzeugkonzeptes auf diese Weise angereizt. Effizientere Motoren, Filter für schädliche Emissionen und mehr aktive und passive Sicherheit wurden erreicht. Aber was passiert, wenn die materiellen und energetischen Voraussetzungen für den fossilen Automobilismus erodieren? Die benötigten Ressourcen sind endlich, ihre Substitution ist unsicher und vielfach mit Nutzungskonkurrenzen verbunden. ${ }^{3}$ Das konventionell betriebene (Privat-)Auto ist kein global verallgemeinerungsfähiges Produkt (vgl. Urry 2012). Eine Motorisierungsrate von 500 bis 800 privaten Autos heutigen Zuschnitts je 1.000 Einwohner in allen oder zumindest in den meisten Ländern der Welt mit dann insgesamt drei bis vier Milliarden Fahrzeugen ist schlichtweg nicht realisierbar. Weder reichen dafür die Brennstoffe noch erlaubt es das Weltklima.

Vor dem Hintergrund dieses unvermeidbaren Spannungsverhältnisses soll im Folgenden eine sozialwissenschaftliche Verortung von Automobilität in Angriff genommen werden. Dabei rückt zunächst der strukturationstheoretische Ansatz von Anthony Giddens ins Zentrum. Darauf aufbauend soll eine erweiterte Perspektive entwickelt werden, die physische (und zunehmend virtuelle) Mobilität als Prinzip der Moderne begreift. Anschließend wird eine Antwort auf die Frage nach Kontinuität oder Bruch des konventionellen automobilen Entwicklungspfades versucht. Am Ende werden Hypothesen zur sozialen Dynamik künftiger Automobilität entwickelt, insbesondere zu den Chancen und Barrieren einer potenziellen automobilen Transformation.

\section{Strukturationstheoretische Annäherungen an den Automobilismus}

Das Auto als eine verbreitete Technik braucht eine aufwändige und extensive Infrastruktur und soziale Verhaltensregeln für die Nutzenden sowie für die anderen Verkehrsteilnehmer. Es bringt Inklusions- und Exklusionseffekte und vieles mehr hervor, was gesellschaftlich wirksam ist oder als Konvention vorausgesetzt wird. Kurzum: es geht nicht um ein isoliertes technisches Artefakt, sondern um ein komplexes Phänomen: den Automobilismus. Im Zuge seines Siegeszuges hat der Automobilismus den Rang einer Institution im Gehlenschen Sinne erlangt und ist damit in den Strudel des wechselseitigen Prozesses von Institutionalisierung und Objektivierung menschlicher Produkte einerseits und einer Internalisierung ihrer Nutzung durch Sozialisation anderseits geraten. Ganz wie Berger und Luckmann das in ihrer Theorie der

2 Mit einzelnen Aspekten der sozialen Normierungs- und Integrationsleistungen im Zuge und in Folge der Automobilisierung haben sich vor Burkart vor allem Claessens (1966) und Sachs (1994) beschäftigt. Zur Synopse früher verkehrssoziologischer Arbeiten siehe Rammler 2001.

3 Bei der stofflichen und energetischen Dimension des Automobilismus geht es sowohl um die für die Herstellung der Fahrzeuge benötigten Materialien und Rohstoffe als auch um die Treibstoffe und Infrastrukturen für ihren Betrieb. Zum Ressourcenverbrauch kommen die Umweltbeeinträchtigungen infolge von Schadstoff- und Lärmemissionen sowie der Flächenverbrauch hinzu. Nur ein Teil dieser volkswirtschaftlichen Kosten ist in den Preisen des Autofahrens internalisiert. Wie hoch die „wahren Kosten“ des Automobilismus sind, ist hoch umstritten, da auch der externe Nutzen in die Gesamtrechnung einzubeziehen ist. Schon hier wird deutlich, dass eine umfassende Betrachtung des Automobilismus der Annäherung aus verschiedenen Disziplinen bedarf. 
Wissenssoziologie entwickeln, ohne vermutlich dabei an das Automobil gedacht zu haben: „Das bedeutet: der Mensch - freilich nicht isoliert, sondern inmitten seiner Kollektivgebilde - und seine gesellschaftliche Welt stehen miteinander in Wechselwirkung. Das Produkt wirkt zurück auf seinen Produzenten“ (Berger / Luckmann 1969: 65).

Diese die Wechselwirkungen zwischen Technik und Individuum betonende Interpretation lässt sich plausibel durch die Giddenssche Theorie der Strukturierung untermauern. Seine beiden zentralen handlungstheoretischen Kategorien, nämlich zum einen die vom Entscheidungsdruck entlastenden Routinen und zum anderen das unbewusste praktische Wissen, erklären insbesondere die alltägliche Nutzung des Automobils. Darüber hinaus sind mit dem Fordismus und Neo-Institutionalismus zwei theoretische Diskurse über den Automobilismus bedeutsam. Diese Diskurse sollen hier jedoch nicht vertieft werden. ${ }^{4}$

\section{Giddens' ,Theorie der Strukturierung “}

So hilfreich fordistische und neo-institutionalistische Erklärungen für die Verbreitung des Automobils auch sind: Seine hohe lebensweltliche Bedeutung aber kann vor allem mit Hilfe der Theorie der Strukturierung von Giddens erklärt werden. Mit seiner Dualität von Handlung und

4 Henry Ford schuf zu Beginn des 20. Jahrhunderts die Grundlagen für einen neuen Modus des kapitalistischen Wirtschaftens, des Konsums und der Vergesellschaftung in den früh industrialisierten Ländern. Im Zuge der Rationalisierungsdynamik der 1920er Jahre wurde Fords Produktionssystem in Deutschland von Friedrich von Gottl-Ottlilienfeld (1925) adaptiert und zur Grundlage einer nationalökonomischen Strategie des Fordismus erhoben. Von einer Massenproduktion von Automobilen konnte zwar zu dem Zeitpunkt in Deutschland und Europa noch keine Rede sein. In den USA allerdings war das Auto seit den 1930er Jahren von paradigmatischer Bedeutung für das fordistische Produktions-, Konsum- und Vergesellschaftungsmodell. Wie die Kleinfamilie, das Eigenheim und der Massentourismus wurde es zum typischen Merkmal der middle-class-society. Doch mit den Krisen von Keynesianismus und Wohlfahrtsstaat, mit der Verschiebung der ökonomischen Gewichte von der industriellen Produktion zu den Dienstleistungen und mit zunehmender sozialer Differenzierung in der Mitte der zweiten Hälfte des 20. Jahrhunderts litt das fordistische Modell als dominantes Akkumulationsund Regulierungsregime erheblich. Doch schon kurze Zeit später erlebten fordistische Prinzipien in einem bisher unbekannten weltweiten Maßstab erneut Triumphe: in den aufstrebenden BRIC-Ländern dominiert die Industrieproduktion die Volkswirtschaften, steigt die Kaufkraft der sich formierenden Mittelschichten und wird von diesen symbolischer Konsum und Wohneigentum angestrebt. Das Auto ist in allen Regionen der beschleunigten nachholenden Modernisierung ein begehrtes Konsumgut. Das westliche Modell steht dabei Pate.

Warum und wie verbreitet sich der Automobilismus als Teil der westlichen Kultur- und Strukturmuster über die ganze Welt? Der neoinstitutionalistische world-polity-Ansatz versteht sich als Erklärungsversuch, ,warum die verschiedenen Gesellschaften in einer nationalstaatlich organisierten Welt einander in vielen überraschenden Hinsichten strukturell ähnlich sind und sich auch in überraschend ähnlicher Weise wandeln" (Meyer et al. 2005: 86). Selbst Globalisierungsgegner, politische Separatisten und religiöse Eiferer berufen sich, wenn auch in Abgrenzung, auf die dominanten globalen Normen der westlichen Leitkultur. Anwendungen ,westlicher Modelle“werden auch in neoinstitutionalistischer Lesart in den seltensten Fällen „eins-zu-eins“ übernommen. Vielmehr entstehen neue Anwendungskontexte durch Rekombination, Hybridisierung und „Fehlkopien“ (Krücken / Haase 2005). Soviel Konvergenz- und Kohärenzbehauptungen blieben nicht ohne Widerspruch. Hinter der world-polityArgumentation verberge sich eine Globalisierungsannahme, wendet Knöbl ein, deren „,theoriebautechnische“ Schwächen nicht zu übersehen seien: „Denn die Behauptung eines weltumspannenden Zusammenhangs beziehungsweise einer stabilen Systemhaftigkeit globaler Interaktionen lässt sich so gut wie nie empirisch belegen" (Knöbl 2007: 208). Meyer und seine Kollegenschaft haben sich mit dem Auto (bisher) nicht beschäftigt. Doch lassen sich mehr oder weniger spekulativ Analogien ziehen: das Auto ist zwar kein universeller Wert an sich, aber es ermöglicht und erleichtert es, diese zu verfolgen. Es stehe in einem engen Wechselverhältnis zu individualistischen Lebensweisen und erweitere die Autonomieräume seiner Nutzer immens. Der Automobilismus ist also ein nützlicher Helfer bei der Realisierung „westlicher Lebensmodelle“. 
Struktur lässt sich begrifflich erfassen, was hier eingangs als sich einander bedingendes symbiotisches Verhältnis von Autoverfügbarkeit und moderner Lebensweise beschrieben wurde. Sie überwindet so die einseitigen Perspektiven, die entweder auf das Individuen und seine Motive und Interessen oder auf die Verhältnisse, die das individuelle Verhalten determinieren, gerichtet sind. So vermittelt sie zwischen der Mikro- und der Makroebene des Automobilismus.

In seiner Theorie der Strukturierung konzentriert sich Giddens auf die Konstitution des Alltagslebens, für die eine eigentümliche Spannung von bewusstem und unbewusstem Handeln gelte. ${ }^{5}$ Er entkleidet den Handlungsbegriff von der üblicherweise unterstellten Intentionalität. Die Subjekte handeln, indem sie ,in die natürliche und soziale Ereigniswelt“ (1988 b: 289) eingreifen. Das tun sie durchaus mit einer bestimmten Absicht, aber nicht ausschließlich und immer. Sie handeln ebenso, ohne einen eindeutigen Zweck zu erfüllen oder ihn explizit machen zu können. Die Grundlage des Handelns ist oft ein intuitives Wissen, das Routinehandeln ermöglicht, ${ }^{6}$ das ,,praktische Bewußtsein“ (Giddens 1988 a: 36).

Giddens argumentiert streng handlungstheoretisch und möchte mit seinem Handlungskonzept dem Problem des Zwangs entkommen, der aus Strukturen erwächst, die dem Einzelnen äußerlich sind. In seinem Konzept handeln Subjekte nicht zwanghaft, da selbst langlebige Institutionen der permanenten Reproduktion durch die Akteure bedürfen. Im Prozess dieser (Re)produktion von Strukturen liegen für die Reproduzierenden demnach durchaus Freiheitsgrade.

Struktur ist also bei Giddens in seinen Worten ,chronisch in das Handeln selbst eingebettet“ (1988 b: 290). Damit ist eine starke Kontingenzvermutung verbunden: ein Akteur kann so, aber auch anders handeln. So bildet Giddens seine Dualität von Struktur, mit der er sich vor allem gegen funktionalistische Theorien absetzt.

Dennoch bleibt das Problem, die Stabilität von Strukturen und das Beharrungsverhalten von Akteuren zu erklären, wenn ständig die Möglichkeit besteht, auch anders zu handeln. In seiner Konzeptionalisierung von Handeln bedient er sich - mit milder Referenz an Heidegger ${ }^{7}$ - einer entwicklungspsychologischen Anleihe: dem ontologischen Bedürfnis nach Sicherheit, das Erik Erikson als Triebkraft für die Entstehung von Vertrauen in die Kontinuität der Ich-Identität ebenso wie in die Konstanz der bekannten Umwelt identifiziert hat. Repetitives Verhalten wie es mit Routinen verbunden ist - sichert Kontinuität und Konstanz. Das ist oft formatiertes und oft gar nicht bewusst regelhaftes Verhalten.

Auf den Automobilismus - den ich als Institution qualifiziert habe - übertragen, bedeutet das Giddenssche Strukturierungskonzept, dass die Autonutzer als Akteure zwar permanent den Automobilismus reproduzieren, dies aber unbewusst-bewusst tun. Autofahren ist kein Zwang, aber andererseits auch nicht immer die Folge absichtsvoller Entscheidungen. Oft wird es nicht bewusst gemacht, vielmehr handelt es sich um ein Nutzen ohne nachzudenken, wie es in Interviews mit Autofahrenden immer wieder zu erkennen ist. Der wichtigste Mechanismus für das Alltagshandeln sind nach Giddens Routinen. Bezugnehmend auf Goffman meint Routinisierung ,de gewohnheitsmäßige, für selbstverständlich hingenommene Natur der großen Masse der Handlungen des Alltagslebens“ (1998 a: 431). Routinen entlasten den Akteur von

5 Handlungstheoretisch setzt sich Giddens in seinem Hauptwerk „Die Konstitution der Gesellschaft“ (1988 a) nicht nur ausdrücklich gegen Parsons ab, sondern nimmt überdies verschiedene psychologische und ethnologische Denkansätze auf (ausführlich dazu: Joas 1992: 207ff).

6 Die entlastende Rolle von Intuition für Entscheidungen generell und ihre paradox erscheinenden Vorteile für rationale Entscheidungen werden auch in der jüngeren psychologischen Debatte hervorgehoben, so prominent von Gigerenzer (2004).

7 Neben der Zeitgeografie von Torsten Hägerstrand und der interaktionistischen Mikrosoziologie Erving Goffmans ist Martin Heideggers Existenzphilosophie einer der „,wichtigsten(n) Bezugspunkte“ in Giddens' Theorie der Strukturierung, wie Markus Schroer hervorhebt (2006: 107). 
Unsicherheit und Entscheidungsdruck, sie laufen ab als Hintergrundprogramme. Und genau für diese psychische Dauerarbeit sind neben Verhaltensroutinen internalisierte Nutzungsformen von Techniken sehr hilfreich. Das Automobil ist eine solche Hilfstechnik und das Autofahren unterstützt das Bewältigen - und Verketten - von unterschiedlichen Aktivitäten an verschiedenen Orten. Gleichzeitig ist das Autofahren höchst voraussetzungsvoll, es setzt Know how, spezifische Fertigkeiten und ein umfangreiches Regelwissen voraus - in Giddens' Sprache: ,praktisches Wissen“. Es erfordert nicht nur formale Qualifikationen wie den Führerschein, sondern auch erfahrungsgesättigte Kompetenzen.

In den Kategorien von Giddens hat das Autofahren als Alltagspraxis starke, aber nicht nur Entlastungswirkungen. Die regelhafte und routinierte Nutzung des Autos mindert existenzielle Unsicherheiten und eröffnet zugleich neue Handlungsspielräume. Hier greift die Kontingenzvermutung in seinem Strukturierungskonzept, mit dem er ausdrücklich ein dezidiert wissenschaftliches Interesse verbindet: „Das zentrale Forschungsfeld der Sozialwissenschaften besteht - der Theorie der Strukturierung zufolge - weder in der Erfahrung des individuellen Akteurs noch in der Existenz irgendeiner gesellschaftlichen Totalität, sondern in den über Zeit und Raum geregelten gesellschaftlichen Praktiken“ (1988 a: 53). Sicherlich schreibt Giddens diese Doppelqualität vielen Alltagspraktiken zu, die Autonutzung ist da kein Sonderfall.

\section{Mobilität als Prinzip der Moderne}

Mobilität kann in einer ersten Annäherung als Beweglichkeit im weitesten Sinne gedeutet werden, wobei der geistige Horizont den Mobilitätsraum markiert. In diesem Mobilitätsraum werden individuelle Handlungsstrategien geplant und auf Realisation geprüft. ${ }^{8}$ Der Mobilitätsraum ist somit als ein Möglichkeitsraum aufzufassen. Mobilität bezeichnet die Bewegung in diesem Möglichkeitsraum (vgl. Bourdieu 1979: 277ff; Giddens 1988 a: $161 \mathrm{ff} ;$ Schulze 1992: 54ff). Dennoch ist dieser Raum zu allererst einmal ein geographischer Bewegungsraum, der physische Bewegung ermöglicht und zugleich begrenzt. Allerdings ist der geografische Raum keine objektiv gegebene Größe. In der jüngeren raumsoziologischen Diskussion wird die konventionelle Raumvorstellung daher zu Recht problematisiert, die den Raum als Container betrachtet. Martina Löw nennt dieses Raumverständnis „,absolutistisch“: „Absolutistisch meint hier, dass Raum als eigene Realität und nicht als Folge menschlichen Handelns gefasst wird. Raum wird als Synonym für Erdboden, Territorium oder Ort verwendet" (Löw 2001: 264). Dagegen plädiert sie für einen „,relationalen Raumbegriff“, der ,,aus der Struktur der Menschen und sozialen Güter heraus abgeleitet wird" (Löw 2001: 264). Diese abstrakte Formulierung reflektiert tief greifende Veränderungen in den Sozialisationsbedingungen moderner Gesellschaften, die unweigerlich auch mit dem Raum und seiner Wahrnehmung zu tun haben. Veränderungen in der Struktur und in der räumlichen Verteilung von Familien, die Verbreitung von Informations- und Kommunikationstechniken und darauf basierende neue Angebote auf dem Markt virtueller Realitäten beispielsweise haben Auswirkungen auf die Raum- und Raumüberwindungserfahrungen nachwachsender Generationen. Beispielsweise erlauben schnelle Verkehrsmittel die Verknüpfung von Räumen, die weit auseinander liegen. Für Scheidungskinder, deren Eltern nach der Trennung an entfernten Orten leben, ist es oft selbstverständlich, über große Entfernungen mit dem Zug oder mit dem Flugzeug zu pendeln. Auch die mediale Präsenz (neuer oder vertrauter) Orte durch Videotelefonie oder webcams beeinflusst die Raumwahrnehmung und lässt Distanzen mental schrumpfen. Der Befund von der „timespace-compression“ (Harvey 1990) verweist darauf. Schließlich eröffnen Simulationspro-

8 Insofern hat die Redewendung „Mobilität beginnt im Kopf“ ihre Berechtigung. Sie zeigt zum einen an, dass die materiellen Voraussetzungen für Beweglichkeit kognitiv erfasst oder nicht erfasst werden, und zum anderen, dass reale Ortsveränderungen mental bereits antizipiert werden. 
gramme Chancen für räumliche Bewegungen, ohne sich überhaupt physisch entfernen zu müssen.

Welche räumlichen Verknüpfungen und Relationen für Bewegungen in Frage kommen und somit angeeignet werden können, hängt auch vom individuellen Informationsstand sowie von der medialen und lebensweltlichen Besetzung und der damit verbundenen kognitiven Repräsentanz von räumlichen Konfigurationen ab. Wie weit sich Mobilitätsräume ausdehnen, ist schließlich durch die Widerständigkeit der Raumüberwindung und das kollektive Wissen darum mitbestimmt. In der angelsächsischen Diskussion werden die Begriffe ,accessibility“ (u.a. Cass et al. 2005) und „motility“ (Kaufmann 2002) verwandt, um zum einen die technischen und infrastrukturellen Gegebenheiten und zum anderen die individuellen Fähigkeiten zu erfassen, Möglichkeitsräume auch nutzen zu können. Gleichzeitig kommt eine Macht- und Herrschaftsdimension hinzu, Räume selbst und Zugänge zu Räumen sind immer gesellschaftlich umkämpft: „Verfügungsmöglichkeiten über Geld, Zeugnis, Rang oder Assoziation sind ausschlaggebend, um (An)Ordnungen durchsetzen zu können, sowie umgekehrt die Verfügungsmöglichkeit über Räume zur Ressource werden kann“ (Löw 2001: 272). Bei allen ungleich verteilten Chancen der Raumverfügung und Raumerschießung ist im Zuge der Modernisierung einst stationärer Gesellschaften jedoch ein generelles Absinken der Raumwiderstände zu beobachten. Was früher ein Privileg nur für wenige war - und das auch noch oft mühsam und langwierig, ist heute für fast alle möglich: die Überwindung auch großer Distanzen. Was noch Ende des 19. Jahrhunderts mit der Kutsche eine Tagesreise war, ist heute mit Bahn, Bus oder Auto in maximal eineinhalb Stunden zu bewältigen. Nicht zu vergessen die durch wirtschaftliche Zwänge motivierte massenhafte Migration von Arbeitskräften über Grenzen und Kontinente hinweg (siehe Mau 2007).

\section{Mobilitätskapital}

Verkehrsinfrastrukturen sind eine Voraussetzung dafür, Raumwiderstände zu überwinden. Erst bei flächendeckend ausgebauten Infrastrukturen können Verkehrsmittel auch effizient genutzt werden. Verkehrsinfrastrukturen sind, ökonomisch betrachtet, zweischneidig, da sie neben externen Kosten wie beispielsweise Lärm, Bodenversiegelung und Landschaftszerstörung gleichzeitig positive externe Effekte hervorbringen. Ökonomen sprechen davon, dass eine umfassende wirtschaftliche Bilanz des Verkehrs neben volkswirtschaftlichen Kosten und Nutzen auch den Optionsnutzen für den Einzelnen berücksichtigen muss (vgl. Eisenkopf 2002). Ein breiter Optionsnutzen kann nur dann entstehen, wenn Verkehrsinfrastrukturen und Verkehrsmittel einfach und möglichst selbsterklärend in der Handhabung sind. Das führt zu einer tendenziell unbegrenzten, damit weltweiten Angleichung von Infrastrukturoberflächen über technische Normen und Standards hinaus. Symbole, Ein-Wort-Bezeichnungen und intuitive Menüführungen lenken die Nutzerin und den Nutzer durch die Verkehrsinfrastrukturen und minimieren damit die Transaktionskosten des Unterwegsseins. Flughäfen sind die Verkehrsorte, deren Benutzungsoberflächen am stärksten vereinheitlicht sind (vgl. Kesselring 2009). ${ }^{9}$

Neben der physischen und räumlichen hat der Möglichkeitsraum auch eine soziale Dimension. Nicht nur die geographischen Gegebenheiten und die technischen und infrastrukturellen Voraussetzungen von Raumüberwindung sind determinierende Faktoren für individuelle Handlungsweisen, sondern auch das jeweilige Vermögen eigene Lebensentwürfe zu erarbeiten und zu realisieren. Hierbei spielen die soziale Positionierung und damit der Zugriff auf geistige

9 Das wird besonders anschaulich in dem Hollywoodfilm Up in the Air, in dem der vielfliegende Hauptdarsteller, gespielt von George Clooney, eine junge Kollegin das optimal vereinfachte Ein- und Auschecken lehrt, ohne in diesen Lektionen des learning by doing zu wissen, auf welchem Flughafen sie sich gerade befinden. 
und materielle Ressourcen sowie kulturelle Dispositionen wie Aufstiegswille oder Bildungsorientierung eine entscheidende Rolle. Mit diesem Verständnis vom Möglichkeitsraum lässt sich an die Sozialstrukturanalyse anknüpfen, die langfristige intergenerative Bewegungen zwischen beruflichen Positionen, Klassenlagen oder Schichten im Verhältnis zu Zugangschancen zu Bildung und Arbeitsmärkten thematisiert (Berger 1996: 157). Weitgehende Einigkeit besteht in der Sozialstrukturforschung darüber, dass neben formalen Bildungsabschlüssen und der Offenheit von Arbeitsmärkten und Karrierewegen auch die individuellen Verkehrsformen und habituellen Ausdrucksfähigkeiten in der Selbstpräsentation im sozialen Raum für einen sozialen Aufstieg entscheidend sind (vgl. im Überblick Berger 2001). Hier wirken kulturelle Barrieren und andere informelle soziale Exklusionsmechanismen sowie nicht zuletzt räumliche Schranken. Den spezifischen Zusammenhang von sozialer Mobilität und geografischem Raum machen Stadtsoziologen wie Häußermann und Siebel stark, wenn sie die fortgesetzte Segregation in Städten als Ausdruck einer Verfestigung sozialer Ungleichheit analysieren (vgl. 1992). Nicht zuletzt verbergen sich geschlechtsspezifische Unterschiede in den Diskursen, die Planungen in der Verkehrsinfrastruktur zugrunde liegen (vgl. Sheller 2008: 258ff).

Bourdieu unterscheidet die zentralen Einflussgrößen auf gesellschaftliche Mobilität als soziales und kulturelles Kapital, die von der Verfügbarkeit über materielle Ressourcen nicht zu trennen sind, aber von diesen nicht determiniert werden (1985). Ihn interessiert, warum soziale Mobilität unter großer Trägheit leidet. Fein gegliederte Verhaltens- und Geschmackscodes formieren einen jeweiligen Habitus (vgl. 1987), der individuelle Habitus dokumentiert gleichsam den Besitz an kulturellem und sozialem Kapital. Kulturelles und soziales Kapital werden vom Einzelnen unter sehr unterschiedlichen Ausgangsbedingungen erworben - oder eben auch nicht. Sie sind das Ergebnis von klassischen, vor allem primären Sozialisationsprozessen. Der Erwerb von kulturellem und sozialem Kapital, die Herausbildung eines spezifischen Habitus, finden zweifellos in überkommenen Klassen- und Schichtenstrukturen statt und dabei spielen räumliche Dichotomien schon immer eine Rolle. Aktivitätenniveaus und Handlungsradien, von denen in der Sozialgeografie so oft die Rede ist, korrelieren historisch offenkundig mit gesellschaftlichem Status und mit der Einkommens- bzw. Vermögenslage. Es gibt viele Hinweise darauf, dass die Schere gesellschaftlicher Stratifizierung desto weiter aufgeht, je ausgedehnter der durchschnittliche räumliche Aktivitätenradius ist. Die Fähigkeit sich in ausgedehnten Räumen erfolgreich und mit verträglichen Transaktionskosten zu bewegen wird damit selbst zu einem wichtigen Bestandteil von kulturellem Kapital. Man könnte, in Anlehnung an Bourdieu, von der spezifischen Form des Mobilitätskapitals sprechen. John Urry verwendet den Begriff des „Netzwerk-Kapitals“ (2007). In beiden Bezeichnungen schwingt das individuelle Vermögen mit, den gesellschaftlichen Rollenerwartungen zu entsprechen und sowohl räumliche als auch soziale Optionen für sich zu nutzen.

\section{Zur Topografie des Möglichkeitsraumes}

Mobilität erfolgt also in einem Möglichkeitsraum, dessen infrastrukturelle bzw. technische und zugleich soziale Ungleichheiten und Ungleichzeitigkeiten eine spezifische Topografie ausbilden. ${ }^{10}$ In den letzten Jahrzehnten haben sich durch die Bildungsexpansion, politische Integrationsprojekte wie die Europäische Union und leichter verfügbare Verkehrsangebote

10 Relevant ist vor allem die Schnittstelle von horizontaler und vertikaler Mobilität, sie markiert zugleich die Chancen gesellschaftlicher Teilhabe. Die Denkfigur einer „Topografie des Möglichkeitsraumes“ erleichtert es, Veränderungen in der Mobilität zu identifizieren und zu veranschaulichen. Sie erleichtert es zugleich Ambivalenzen zu erkennen und zu beschreiben. Zu den Anfängen dieser Denkfigur siehe Canzler / Knie 1998: 25ff. 
grundsätzlich die Zugänge zwar erweitert. Dies muss jedoch nicht in jedem Fall einen Zuwachs von kollektivem Mobilitätskapital bedeuten. Ausdehnungen können auch zu bloßen räumlichen Erstreckungen und - im doppelten Sinne - zur Verflachung der Topographie des Möglichkeitsraumes führen. ${ }^{11}$ Es bietet sich also an, begrifflich zwischen Verkehr und Mobilität zu unterscheiden: Verkehr ist zu verstehen als Bewegung in konkreten Räumen, während Mobilität Bewegung in möglichen Räumen ist. Vieles, was mit Mobilität bezeichnet wird, ist in dieser Lesart nichts anderes als Verkehr.

Eine begriffliche Trennung zwischen Mobilität und Verkehr - und analog von motility bzw. Mobilitätskapital und accessibility - hat auch Vorteile für die empirische Forschung zum Verkehrsverhalten. So kann die Ermittlung der täglichen Wege oder der gefahrenen Kilometer, also die klassischen Kennzahlen des individuellen Verkehrsaufwandes, ${ }^{12}$ von der Frage getrennt werden, ob eine Person mit hohem Verkehrsaufwand auch mobiler ist als eine Person, deren Wegezahl geringer oder deren Wegestrecken kürzer ausfallen. Ambivalenzen können präziser erfasst werden. Eine bloße Erweiterung des geografischen Möglichkeitsraumes, d. h. die Überwindung größerer Entfernungen, ist nämlich kein Garant für eine entsprechende Erhöhung der Mobilität. Eine eingeschränkte motility kann sogar zu höherem zirkulärem Verkehrsaufwand führen, weil keine Möglichkeiten bestehen oder bestehende Optionen nicht wahrgenommen werden, spezifische Bedürfnisse im Nahraum zu befriedigen. Eine höhere Wegezahl oder größere Wegelängen können umgekehrt sogar ein Weniger an Mobilität bedeuten. Für Berufspendler beispielsweise wird der werktäglich zu bewältigende Weg zur und von der Arbeit zum Transitraum, seine Bewältigung ist eine einfache (und manchmal doch nur mühselige) Distanzüberwindung, der persönliche Möglichkeitsraum wird keineswegs größer. Er wird sogar eingeschränkt, weil das Pendeln starre Abfahrtszeiten vorgibt oder Staus bzw. Verkehrsblockaden den Zeitdruck zusätzlich erhöhen.

Während für die Erfassung der Verkehrsströme ein ausdifferenziertes, wenn auch immer noch verbesserungsfähiges Messinstrumentarium zur Verfügung steht, stellt sich die Situation bei der quantitativen Bestimmung individueller Topographien von Möglichkeitsräumen weitaus schwieriger dar. Auch für die Vermessung der sozialen Mobilität kann auf eine lange Theorie- und Methodendiskussion bei der Sozialstrukturanalyse Bezug genommen werden (vgl. im Überblick Solga et al. 2009). Doch gibt es keine etablierten brauchbaren Indikatoren für die Beschreibung sowohl von Mobilität im Sinne der Erweiterung von individuellen Möglichkeitsräumen als auch von motility als Fähigkeit sich angemessen und erfolgreich im Raum zu bewegen. Bildungsstand und Einkommensniveau sind gängige sozialindikative Kategorien. Sie tragen maßgeblich, wenn auch nicht ausschließlich, zur Bildung des individuell inkorporierten kulturellen Kapitals bei. Insofern können sie als Näherungswerte für den Stand und die durchschnittliche Verbreitung von Mobilitätskapital eingesetzt werden. Für seine exakte Bestimmung gibt es jedoch keine eindeutigen Kenngrößen, weder für das Individuum noch auf der Ebene des Haushaltes und ganz zu schweigen von der Gesellschaft als ganzer. Die Ver-

11 Verflachung ist das Stichwort für ein ganzes Genre kulturpessimistischer Publikationen, das vor den negativen Folgen der vielfältigen Mobilitätserweiterungen warnt. Neben Sennetts essentialistischer Analyse einer angeblich zerstörerischen Flexibilisierung aller Arbeits- und Lebensverhältnisse (1998) thematisieren im deutschsprachigen Raum beispielsweise Geißler (1998) und Reheis (1998) den Zusammenhang von Bescheunigung, Entwertung von Raum und ökologischen Folgeschäden. Die stärkste intellektuelle Schubkraft dürfte Rosa mit seiner Beschleunigungsthese entfaltet haben (2005).

12 Von dem der Verkehrsforscher und -planer Eckhard Kutter zurecht lieber spricht als von Verkehrsleistung, was ihm zu euphemistisch klingt (vgl. 1975). 
wendung des Begriffs des Möglichkeitsraumes hat somit ebenso wie der motility-Ansatz einen vornehmlich heuristischen Wert. ${ }^{13}$

\section{Kontinuität oder Bruch}

Ein Mehr an Mobilität und an gesellschaftlichen Handlungsoptionen ist auch das Ergebnis eines besseren Verkehrsangebots, vor allem in der Folge der Massenmotorisierung. Sowohl territoriale Grenzen als auch zeitliche Standardregime und soziale Privilegien sind durch die Ausweitung von Möglichkeitsräumen löchrig geworden. Die individuellen Möglichkeitsräume erweiterten sich permanent und in alle Richtungen. Die Globalisierung dehnt den bestehenden Trend über bisherige nationale und eingespielte sozialräumliche Verflechtungen aus, sie geht mit einer signifikanten Ausweitung der Möglichkeitsräume für mehr Menschen als je zuvor einher.

Das ist eine verzerrte Sicht der Dinge, so möchte man einwerfen. Der Stau, die extreme Verengung aller individuellen Möglichkeitsräume, ist schließlich nicht nur zum Sinnbild des modernen Verkehrs geworden, sondern in vielen Ballungszentren alltägliche Realität. Und werden in den bisweilen euphorischen Hymnen auf die Globalisierung die weiten und transnationalen Distanzen nicht weit überschätzt? Verkehrszählungen zeigen, dass fast 90 Prozent des Verkehrsaufwandes im Nahbereich der Verkehrsteilnehmer geleistet wird (siehe Kutter 2007: 254-255). Zugleich wird der Personenfernverkehr von einer begrenzten Anzahl von Vielfahrenden dominiert, zu der allerdings mit zunehmender Tendenz auch abhängig Beschäftigte gehören, zu deren Aufgabenprofil eine hohe Reisetätigkeit gehört, worauf Kesselring und Vogl hingewiesen haben (2010). Sieht man von Urlaubsreisen ab, die sich gleichmäßiger auf alle Einkommensklassen und Bildungsabschlüsse verteilen, sind sonstige Fernreisen auf einen kleinen hochmobilen Bevölkerungsanteil beschränkt. Die Hochmobilen machen sowohl viele Dienstreisen als auch überdurchschnittlich oft zusätzliche lange private Reisen. ${ }^{14}$ Nicht nur der Zugang zu den Verkehrsmitteln, die accessibility, ist höchst unterschiedlich, auch die Kompetenzen, diese effizient zu nutzen, die motility des einzelnen Verkehrsteilnehmers, sind ungleich ausgeprägt. Eine erweiterte Mobilität kennt Gewinner und Verlierer, Mobilitätschancen sind keineswegs gleich verteilt. ${ }^{15}$

Doch auch die vermeintlichen Gewinner, die mehr-Mobilen, sind nicht vollkommen frei in der Gestaltung ihrer Mobilität. Denn erweiterte Mobilitätschancen erhöhen den sozialen Druck, diese auch zu nutzen. Das Auto spielt hierbei wiederum eine Schlüsselrolle. Die tech-

13 In den letzten Jahren haben Kay Axhausen und seine Mitarbeiter den Versuch gemacht, über Wegemodellierungen den individuellen Möglichkeitsraum zu vermessen und grafisch darzustellen. Dies führte zu Abbildungen von Aktionsradien und Wegehäufigkeiten und veranschaulichte vor allem, wie unterschiedlich die Bewegungsprofile innerhalb der untersuchten Gruppe sind. Die Qualität der Wege, sei es der Routinegrad, die Freiheiten in der Gestaltung bzw. im zeitlichen Ablauf etc., lasse sich so jedoch nicht erfassen, dafür bedürfe es dann wiederum qualitativer Interviews (vgl. Ohnmacht et al. 2008; Larsen et al. 2006).

14 Das zeigt eine repräsentative Befragung zu Fernreisen in Deutschland im Rahmen des Forschungsvorhabens Die intermodale Vernetzung von Personenverkehrsmitteln unter Berücksichtigung der Nutzerbedürfnisse, INVERMO. Dort heißt es im Schlussbericht zusammenfassend: „Überschlägig lässt sich festhalten, das fast $50 \%$ der Fernreisen von etwa $10 \%$ der Bevölkerung durchgeführt werden, während andererseits etwa die Hälfte der Bevölkerung nur etwa $10 \%$ der Fernreisen unternimmt. Etwa $14 \%$ der Bevölkerung muss als im Fernverkehr nicht aktiv (,immobil“) gelten, sie unternehmen im Jahresverlauf keine Reisen mit mehr als 100 km einfache Fahrstrecke“ (Zumkeller et al. 2005: 82).

15 Diese Dimension der sozialen Ungleichheit ist in der Ungleichheitsforschung unterbelichtet. Während die Ausstattung mit materiellen Ressourcen, die soziale Herkunft, die Bildungschancen oder die geschlechtsspezifische Rollenzuweisung als Gründe für soziale Ungleichheit und ihre Reproduktion im Blick sind (vgl. Solga et al. 2009), ist motility keine Erklärungsgröße. So erstaunt es nicht, dass es auch keine Indikatoren oder Kennzahlen gibt, mit denen sie erfasst und gemessen werden kann. 
nischen Mobilitätsoptionen infolge der Massenmotorisierung sind zum allgemeinen Standard und zur Basis sozial erwünschter Flexibilität geworden. John Urry hat diese Ambivalenz der Automobilität pointiert beschrieben: „Automobility is a Frankenstein-created monster, extending the individual into realms of freedom and flexibility whereby inhabiting the car can be positively viewed and energetically campaigned and fought for, but also constraining car,users' to live their lives in spatially stretched and time-compressed ways. The car is the literal,iron cage' of modernity, moving and domestic" (2004: 28). Automobile Möglichkeitsräume werden zu sozialen Verpflichtungsräumen, sobald sie eine bestimmte Verbreitung gefunden haben.

\section{Opfer des eigenen Erfolges}

Die Kritik am Auto, vor allem in Form einer Skandalisierung seiner nicht-intendierten Nebenfolgen, hat es im Laufe seiner Erfolgsgeschichte zu keinem Zeitpunkt geschafft, eine wirkliche Bedrohung für die Attraktivität des Autos oder für die Legitimität der Autoindustrie zu werden. Vielmehr hat sie ebenso wie die gesetzlichen Umwelt- und Sicherheitsauflagen die Adaptionsfähigkeiten der Hersteller erhöht und eine Reihe von technischen Optimierungen beflügelt. Zur Bedrohung des Automobils könnte allerdings und paradoxerweise gerade sein Erfolg werden. ${ }^{16}$ Das rasante, in Folge der weltweiten Finanz- und Wirtschaftskrise 2008/9 nur vorübergehend gedrosselte Wachstum der weltweiten Autoflotte unterminiert in zweierlei Hinsicht die eigene Basis: zum einen schränken Staus und Parkplatzprobleme die automobile Autonomie erheblich ein. Eine hohe Fahrzeugdichte und ein auf die potenziellen Kapazitäten bezogen niedriger Auslastungsgrad der einzelnen Gefährte bedeuten einen Flächenbedarf, der in Agglomerationsräumen zu Konkurrenzen um knappen Raum führt. Das gilt besonders für die Mega-Cities Asiens und Südamerikas, in denen der Zuwanderungsdruck immens und die nachholende Motorisierung in vollem Gange ist. Zum anderen führt der weltweite Anstieg des Autoverkehrs zu einem Mehrverbrauch und damit zu einer beschleunigten Erschöpfung der begrenzten fossilen Energien. Der als peak oil bezeichnete Scheitelpunkt der Ölausbeute ist zwar schwer zu bestimmen, aber allein seine Wahrscheinlichkeit bedeutet unter dem Vorzeichen einer steigenden Ölnachfrage, dass der Markt volatiler wird.

Mehr als ein Jahrhundert jedoch ist die Zahl der Autos ununterbrochen und in den letzten Jahrzehnten sogar drastisch gewachsen. Sein Wachstum hat zur Omnipräsenz des Automobils geführt. Ist das Auto verfügbar, entfaltet es seinen Aufforderungscharakter zur häufigen und in der Folge routinemäßigen Nutzung. Techniksoziologisch betrachtet lässt sich die Verbreitung und hohe Verfügbarkeit des Autos in den früh industrialisierten Ländern so zuspitzen: Es schreibt sich in Alltagspraktiken ein (Rammert 1993) und erlangt einen Akteursstatus im Sinne von Bruno Latour $(1996,2005)$. Autoaffine Lebensweisen und Siedlungsstrukturen bilden sich aus, diese fördern „entfernungsintensive Lebensstile“ (Holzapfel 1997). Schließlich ist der Automobilismus Ausdruck einer auf soziale Distinktion zielenden individualistischen Konsumweise, die auf der doppelten materiellen Basis einer industriellen Massenproduktion und leicht verfügbarer fossiler Energien beruht.

16 Dieser paradoxe Gedanke, dass nämlich das Auto mit seiner Verbreitung die Voraussetzungen seines Erfolgs aufzehrt, findet sich analog in der These von Burkard Lutz (1984) zur Erfolgsgeschichte des westlichen industriell-kapitalistischen Wohlstandsmodells nach dem Zweiten Weltkrieg, die ihr Ende in der Erschöpfung der „,kulturellen“ Ressourcen vorkapitalistischer Sozialisation in der Mitte der zweiten Hälfte des 20. Jahrhunderts gefunden habe. 
Es mutet seltsam an, aber trotz Staurekorde und vieler negativer weiterer Folgen der Massenmotorisierung gibt es kaum eine radikal-ablehnende Autokritik. ${ }^{17}$ Auch unter kritischen Verkehrswissenschaftlern hat sich die Position verbreitet, dass die Attraktivität des öffentlichen und des nicht-motorisierten Individual-Verkehrs nicht gegen, sondern nur mit dem Auto zu verbessern ist. Konzepte zur Gestaltung einer attraktiven Kooperation zwischen Automobilität und Öffentlichem Verkehr liegen vor (siehe Canzler et al. 2007; Schade et al. 2011). ${ }^{18}$

Die Geschichte des Automobils der letzten Jahrzehnte ist geprägt von Phasen des Aufbruchs und der Anpassung an externe Ansprüche und regulatorische Auflagen einerseits und von Phasen des business as usual andererseits. In den Krisenjahren 2008/09 hat eine neue Phase des Aufbruchs begonnen. Vom „Strukturwandel im Automobilsektor" war mal wieder die Rede (FES 2010: 36), die Frage „Automobility in Transition?“" (Geels et al. 2011) wurde gestellt. Tatsächlich, die Hersteller beschlossen downsizing-Strategien für ihre konventionellen Modelle, sie setzten Flottenversuche mit Elektroautos um und sie zeigten sich offen für alternative Energieträger. Vom green car ist seither auf Automessen und auf den Motorseiten der Zeitungen oftmals die Rede. Noch ist nicht entschieden, ob diese Öffnungsphase schnell vorbei sein wird oder ob bereits ein Paradigmenwechsel zu einer postkonventionellen - und das heißt zu einer postfossilen - Automobilität begonnen hat (vgl. Canzler / Marz 2011). Denkbar ist ebenso eine - vielleicht temporäre - Überlagerung von fossilen und postfossilen Entwicklungspfaden. ${ }^{19}$

\section{Raumwiderstände sinken nicht mehr}

Die Ausweitung von Möglichkeitsräumen für zunehmend mehr Menschen beruht seit Jahrzehnten darauf, dass die Verkehrskosten relativ zum verfügbaren Einkommen sinken und zugleich die Nutzungsoberflächen aller Verkehrsmittel, nicht nur beim Auto, sich sukzessive angleichen und damit mentale Hürden einer Nutzung niedriger werden oder ganz verschwinden. In dieser Verbilligung und Vereinfachung von Transport und Verkehr lag - und liegt noch - eine Voraussetzung für die Ausweitung und für die räumliche Vernetzung in der Produktion, in der Distribution und ebenso im Konsum. ${ }^{20}$ Doch hängen diese technischen und wirtschaftlichen Entwicklungen eng mit sozialen zusammen: Aufgrund gewachsener kollektiver Mög-

17 Zu den wenigen radikalen Autokritikern gehören der österreichische Verkehrsplaner Hermann Knoflacher, der polemisch gegen das Auto als „Irrläufer der Evolution“ wettert (siehe jüngst: Knoflacher 2009), und der Technikkritiker Otto Ullrich (1988).

18 Erste Versuche einer Annäherung unter dem Label Intermodale Verkehrsdienstleistungen werden gemacht. In metropolitanen Regionen werden Mietfahrradsysteme eingeführt und damit die Optionen für die Nutzer des Öffentlichen Verkehrs erweitert. Nachdem in einigen dicht bebauten europäischen Metropolen das öffentliche Mietrad (und in seinem Windschatten auch das private Rad) eine viel genutzte Ergänzung des public transport geworden ist und derzeit auch außereuropäische Städte die Einführung von public bike-Systemen vorantreiben, werden bereits public car-Systeme geplant oder schon eingeführt.

19 Das mehrere Jahrzehnte gültige Entwicklungsmuster des ,größer, schneller, schwerer und teurer“ hat Konkurrenz bekommen, neue Billigmarken mit überdurchschnittlichen Zuwachsraten wie die Renault-Tochter Dacia haben bereits vor der Finanz- und Wirtschaftskrise die etablierten Anbieter in Unruhe versetzt. Eine Differenzierung in den Typen und Größenklassen bzw. Ausstattungsmerkmalen geht mit einem weltweiten Wachstum des Automobilmarktes einher. Die Hersteller sind unsicher, welche Marktsegmente sie zukünftig bedienen müssen. Zugleich wurden die Produktionskapazitäten ausgeweitet. Die Gefahren einer zunehmenden „Rendite-Squeeze“ steigen (Nieuwenhuis / Wells 2008).

20 Die Implikationen günstiger Transportkosten, oder abstrakter formuliert: die Effekte abnehmender Raumwiderstände, sind weitreichend und vielfältig. So sind sie beispielsweise Voraussetzung für das Einkaufen auf der grünen Wiese, für das (Fern)pendeln, für die expandierende Eventkultur und für den boomenden Kurzzeit- und Städtetourismus. 
lichkeitsräume sind die sozialen Erwartungen an die oder den Einzelnen gestiegen. Souveränitätsgewinne und Zwangsverkehre liegen eng beieinander: das suburbane Reihenhäuschen mit Erst- und Zweitwagen ist ein solcher Fall.

Das billige Öl war „Schmiermittel“ des ökonomischen Aufstiegs der OECD-Welt in der zweiten Hälfte des 20. Jahrhunderts. Es hat die Produktions- und Konsumformen geprägt und zugleich eine gesellschaftliche Erwartungshaltung befördert, dass der individuelle Möglichkeitsraum größer und größer wird. Ist diese Ära des billigen Öls an ihr Ende gekommen und drohen zugleich höhere Kosten in Folge einer aktiven Bekämpfung der Folgen des Klimawandels, hat das Auswirkungen auf den Verkehr ebenso wie auf Wohn- und Siedlungsweisen, auf die Berufsmobilität und auf das Freizeit- und Urlaubsverhalten. Voraussichtlich werden einige nahe liegende Kompensations- und Optimierungsschritte erfolgen, ohne dass die Mobilitätsmuster sich grundlegend ändern: verbrauchsärmere (und/oder billigere) Fahrzeuge werden eine größere Rolle spielen, einige Wege werden gebündelt, einzelne Spritztouren nicht unternommen. Möglich ist zudem, dass sich die noch schwache Tendenz einer Rückkehr in die Stadt verstärken wird und damit eine ressourcenschonende Verdichtung der Siedlungsstrukturen eintritt. Offen ist, ob sich regionale Produktionsstrukturen und ein stärker regional orientierter Konsum verbreiten und ob sich die Berufs- und Bildungsmobilität dahingehend verändert, dass die Beschäftigten verstärkt den Arbeitsplätzen hinterherziehen.

Die direkten und kurzfristigen Auswirkungen von aktiver Klimaschutzpolitik, überdurchschnittlich steigenden Ölpreisen, knappen Verkehrsflächen in Ballungsräumen sowie sozialer Desintegration auf die (auto)mobile Gesellschaft sind bisher wenig spektakulär. Da es sich jedoch um anhaltende Entwicklungen mit teilweise sogar sich verschärfender Dynamik handelt, haben sie längerfristige Konsequenzen mit dem möglichen Effekt hoher sozialer Selektivität. Überdurchschnittlich steigende Mobilitätskosten treffen in erster Linie die Einkommensschwachen. Die erreichte gesellschaftliche Teilhabe breiter Bevölkerungskreise, insbesondere Freizeit- und Urlaubsmöglichkeiten, die sich in der Zeit billigen Verkehrs für viele eröffnet hatten, sind gefährdet. Nicht nur, dass damit verbundene Märkte wegbrechen. Ferne könnte wieder zu einem Privileg und Fernreisen stärker als bisher schon zu einem sozialen Distinktionsmerkmal werden.

\section{Zur sozialen Dynamik künftiger Automobilität}

Angesichts fortgesetzter Urbanisierung wird der Platz in den Städten zunehmend zum Engpass. Raumkonkurrenzen bedrängen das private Auto, sein Platzbedarf macht es kostspielig. Das private Auto wird unter diesen Bedingungen (wieder) zum Luxusgut. Während die fixen Kosten des Automobils stagnieren oder nur leicht steigen, ist mit einer erheblichen Zunahme der variablen Kosten seiner Nutzung zu rechnen. ${ }^{21}$ Das ist vor allem dann zu erwarten, wenn Raumkonkurrenzen in städtischen Agglomerationen und die Erschöpfung der fossilen Energiebasis einen aufwändigen Umbau des Mobilitätsangebotes erzwingen.

\section{Exklusionseffekte der Transformation des Automobilismus}

Überdurchschnittliche Kostensteigerungen im Verkehr wirken sozial selektiv. Es ist fraglich, ob das Versprechen der gesellschaftlichen Teilhabe und der sozialen Mobilität, das über Jahrzehnte an die Senkung des Raumwiderstandes gebunden war, auch zukünftig noch aufrechterhalten werden kann. Die Sicherung sozialer Teilhabe war in fordistischen Zeiten an ver-

21 Das ist nicht erst in Zukunft so. Bereits in den Jahren 2005 bis 2011 sind die Treibstoffpreise in Deutschland um 28 Prozent gestiegen, während sich die allgemeinen Lebenshaltungskosten lediglich um 11,1 Prozent erhöht haben (vgl. Destatis 2012). 
gleichsweise günstige Energie- und Rohstoffpreise sowie an eine Externalisierung der Umweltkosten geknüpft. Angesichts von Ressourcenknappheiten und steigendem Internalisierungsdruck drohen neue Formen der sozialen Spaltung. Auch können sich sozialräumliche Disparitäten vergrößern.

Insgesamt ist also damit zu rechnen, dass Mobilität im Zeichen der Dekarbonisierung der Produktions-, Distributions- und Konsumtionsprozesse (wieder) zu einer sozialen Frage wird. Zunächst wird vermutlich die Nachfrage nach kostengünstigen Verkehrsangeboten zunehmen. Steigen die variablen Kosten des Autofahrens, dürfte der Druck auf die fixen Kosten steigen und damit das low cost-Segment stärker werden. Insbesondere Anbieter aus China und Indien werden voraussichtlich in diesen Markt drängen. Auch im öffentlichen (Fern-)Verkehr werden mehr kostengünstige Angebote entstehen, beispielsweise beim Fernbusverkehr. Zum anderen werden alle Optionen an Bedeutung gewinnen, die eine bessere Auslastung von Autos versprechen. Das sind nicht zuletzt private Fahrgemeinschaften, gewerbliche Mitfahrzentralen, online-per-Anhalter-Trips etc. Das Carsharing und der öffentliche Nahverkehr können ebenfalls profitieren, allerdings ist auch hier mit überdurchschnittlichen Kosten- und damit Preiserhöhungen zu rechnen. Wenn die Raumüberwindung mit höheren Kosten verbunden ist, könnte es darüber hinaus zu einer vermehrten Virtualisierung physischen Verkehrs kommen.

Rückwirkungen des Endes des billigen Verkehrs und des sich erhöhenden Raumwiderstandes sind schließlich auch auf die soziale Mobilität zu erwarten. Zumindest waren historisch physische und soziale Mobilität eng miteinander verbunden. Sozialer Aufstieg hat mit Bildung und Wissen, aber auch mit der Bereitschaft und der Möglichkeit zu tun, den Ort zu wechseln und somit den eigenen Horizont zu erweitern. Der Arbeitsmarkt ist längst ein überregionaler, Berufschancen und Ausbildungsstätten sind im nationalen und zunehmend im internationalen Raum verteilt. Umgekehrt sind die Arbeitsmarktsektoren am gefährdetsten, deren potenzielle Beschäftigte am unflexibelsten, d.h. am wenigsten mobil sind.

Tangiert ist schließlich die kulturell-symbolische Dimension von Autobesitz und Autonutzung. Parallel zur Heterogenisierung des Angebotes und zur Differenzierung bzw. Segmentierung der Nachfrage scheint sich eine Veränderung in den Einstellungen zur Automobilität abzuzeichnen. Der Führerscheinbesitz bei den unter 26jährigen ist laut dem Deutschen Mobilitätspanel erstmals seit vielen Jahren signifikant gesunken (vgl. Zumkeller et al. 2010: 17). Gleichzeitig stieg der Anteil des öffentlichen Verkehrs am modal split der Jungen bis Mitte 20 deutlich an (im Überblick siehe: Tully 2011). Abnehmenden Pkw-Zulassungszahlen in dieser Altersgruppe ergänzen das Bild. ${ }^{22}$ Ein weiteres Indiz für die Erosion der automobilen Hegemonie ist die Verschiebung der modal split-Anteile zum Fahrrad (vgl. DIW / Infas 2009 und Ahrends 2009) und die Erfolge von public-bike-Angeboten in vielen Städten in Deutschland und europaweit (vgl. Matthies 2009). Der Fahrradanteil am Verkehrsaufkommen hat in erster Linie dort stark zugenommen, wo er bislang gering war. Außerdem hat sich herausgestellt,

22 Das ist nicht nur in Deutschland zu beobachten, sondern auch in anderen Ländern der OECD-Welt. Länder mit noch höherer Motorisierung wie Norwegen vermelden ebenfalls hohe Rückgänge bei Führerscheinbesitz und Fahrleistungen Jüngerer (vgl. Ifmo 2011). Daraus eine generelle Abwendung der jüngeren Generationen vom Auto abzuleiten ist allerdings nicht zulässig. Skepsis an der Repräsentativität und an der Reichweite dieser Aussage diverser Auto-und-Jugend-Studien, die oft von Unternehmensberatungen stammen und auf sekundären Inhaltsanalysen beruhen, ist angebracht (vgl. Rust 2011: 66ff). Zumindest gilt die alte Formel ,Je größer das Auto, desto größer der Prestigegewinn“ nicht mehr. Es gibt sogar Anzeichen dafür, dass diese Formel kippt und es umgekehrt vielmehr der Rechtfertigung bedarf, ein großes, schweres und übermotorisiertes Auto zu fahren. Vor allem die Jüngeren entwickeln ein offenbar selbstverständliches Verhältnis zum Auto: für sie ist das Auto zwar ein beliebtes Verkehrsmittel, jedoch weniger ein Status- und Prestigeobjekt als für die Vorgängergenerationen (vgl. Kraftfahrtbundesamt 2010; Tully 2011). 
dass das Fahrrad signifikant häufiger als das Auto mit anderen Verkehrsmitteln kombiniert wird (vgl. Zumkeller et al. 2010: 62).

Schwächt sich die starke Kopplung von modernen Lebensformen und (privater) Automobilität ab? Sind postautomobile Vergemeinschaftsmuster erkennbar, die an die Stelle des individuellen Besitzes treten? Angesichts der über Generationen eingeübten „Wahlverwandtschaft von Mobilität und Moderne“"(Rammler 2011) spricht zunächst viel gegen eine Prognose, dass automobile Lebensweisen erodieren und dass die hohe Affinität moderner, intentional selbstbestimmter Lebensentwürfe gegenüber dem Automobil abnimmt. Selbstbeweglichkeit ist ein essentielles Merkmal moderner arbeitsteiliger Lebensweisen und wurde in der zweiten Hälfte des 20. Jahrhunderts zunehmend mit dem Auto realisiert, das nach dem Konzept des Universalfahrzeugs entwickelt und massenhaft nachgefragt wurde. Es sind die automobilen Routinen in der Alltagspraxis, die es allen Alternativen zum privaten Auto bisher so schwer gemacht haben. Diese Routinehandlungen sind es, die nach Giddens ,ein Gefühl der Seinsgewißheit sowohl fördern wie umgekehrt in diesem auch ihren Rückhalt finden“"(1988 a: 431).

Das jederzeit verfügbare Auto ist für den individuellen Nutzer das ideale Verkehrsmittel für eine komplexe Alltagsorganisation. Es entlastet von zusätzlichen Koordinierungsaufgaben für ständig wiederkehrende Aktivitäten und bietet zugleich das Maß an Flexibilität, das individuell gewünscht und zugleich gesellschaftlich erwartet wird. Für das private Auto gibt es kein funktionales Äquivalent. Der Öffentliche Verkehr und auch ein um ein flexibles Leasing erweitertes Carsharing haben seine Position - zumindest bisher - nicht einnehmen können. Allerdings ändert sich der Kontext urbaner Mobilität wesentlich, Informations- und Kommunikationstechniken wie dem mobilen Internet, WLan und leistungsfähigen Smartphones kommt dabei eine Schlüsselrolle zu. Ihre Diffusion modifiziert nicht zuletzt die Beziehungen zwischen nichtAnwesenden, also die Kopräsenz, wie sie Giddens als zentrale Kategorie seiner Strukturierungstheorie eingeführt hat. Anwesenheit im körperlichen Sinne ist weniger denn je nötig, um zu kommunizieren. Online zu sein ist eine zusätzliche und bedeutsamer werdende Variante von Kopräsenz. Im Zuge einer permanenten online-Anbindung entstehen zudem neue Verhaltensmuster. Emails werden permanent abgerufen, die Nachrichtenlage in sozialen Netzwerken wird ständig aktualisiert. Dazu gehören außerdem Abfragen bei Nachrichtenportalen, Wetterdiensten oder Börsen. Routinemäßige temporäre Kopräsenzen werden zunehmend handlungsrelevant. Unter diesen Bedingungen können auch netzbasierte und auf Bildschirmen repräsentierte Mobilitätsdienstleistungen selbstverständlich und Teil des Alltagshandelns werden.

Die These vom Bedeutungsverlust des privaten Automobils lässt sich also durchaus techniksoziologisch begründen: die Durchdringung des Alltags mit polyvalenten Informationsund Kommunikationstechniken untergräbt das Monopol des Automobils in Bezug auf Selbstbeweglichkeit im Verkehr. Sie ermöglicht neue Mobilitätsangebote und macht sie ihrerseits routinefähig. Empirische Belege für diese These sind allerdings noch spärlich, sie sind bislang vor allem bei den digital sozialisierten Jüngeren zu finden, die sich im Modus einer IuKvermittelten Raumerschließung und -überwindung in urbanen Räumen bewegen (vgl. Tully / 
Alvaraz 2010). Sie sind es gewohnt, in vielen Handlungsbereichen mit Zugriffsrechten zu agieren. $^{23}$

Im Gegensatz zum bisherigen Trend, wonach die Hindernisse im Raum für Menschen, Waren und Informationen sukzessive kleiner geworden sind, sind mit der Heterogenisierung von Automobilität neue Hindernisse zu erwarten. Bisher ging die Tendenz bei den Nutzungsoberflächen von Verkehrsmitteln zur Vereinfachung durch Vereinheitlichung. Das Design und die Organisation von Flughäfen veranschaulichen diese Tendenz. Verkehrsschilder und Verkehrstelematik unterliegen ebenfalls einem Druck zur Vereinheitlichung. Dieses Ziel kann zukünftig in Konflikt mit einer Entwicklung zu mehr und vielfältigen Verkehrsangeboten und -einschränkungen vor Ort geraten. Ein wichtiger Treiber für eine solche Heterogenisierung der formalisierten Regeln von Automobilität ist die bislang noch zaghaft eingeführte, aber von den Verkehrswissenschaften unisono eingeforderte Nutzerfinanzierung im Verkehr. Das zugrunde liegende ökonomische Prinzip ist simpel: Nutzer sollen für die Inanspruchnahme von Verkehrsinfrastruktur zahlen. Konsequent umgesetzt würden zum einen neben den Betreiberkosten auch bisher externe Kosten in die Nutzungsgebühren Eingang finden und zum anderen würden Nutzungsentgelte in Abhängigkeit vom Verkehrsaufkommen variieren. Verkehrsinfrastrukturen verlieren mit der Umstellung auf ein System der Nutzerfinanzierung ihren Charakter als öffentliche Güter, dafür gibt es gute verkehrswirtschaftliche und ökologische Gründe. Die Folgen für gesellschaftliche Partizipationschancen sind jedoch problematisch: die freie Wahl von Wegen und Fahrtzeiten ist mehr denn je abhängig vom verfügbaren Einkommen. Soziale Unterschiede schlagen sich unter Bedingungen der konsequenten Nutzerfinanzierung auf die Mobilitätschancen nieder, Mobilität ist damit ein zusätzlicher Faktor für eine sich verschärfende soziale Exklusion einkommensschwacher Schichten. Die Fähigkeit zur Mobilität, in der Terminologie der sozialwissenschaftlichen Mobilitätsforschung: die „motility“ (vgl. Kaufmann 2002), wird wichtiger.

Motility hat in diesem Zukunftsszenario einer stärker (re)regulierten (Auto)Mobilität eine materielle Seite, nämlich die Chance und Bereitschaft, sich Mobilität und damit soziale Teilhabe unter erschwerten Bedingungen leisten zu können. Sie hat zugleich eine kognitive bzw. mentale Seite: Sie hängt ab vom Wissen um Mobilitätsangebote und auch von der Fähigkeit, Alternativen abwägen zu können, sowie von der Bereitschaft und Selbstsicherheit, Neues auszuprobieren und Unsicherheiten auszuhalten. Was Giddens generell als „diskursives“ und „praktisches“ Wissen unterscheidet und zugleich als komplementäres Gegenüber der objektiven Strukturen konstruiert (1988 a: 291), macht ebenso motility aus. Die individuellen Möglichkeiten, dieses Wissen auf- und auszubauen, sind jedoch ungleich verteilt. Sie korrelieren - ähnlich wie die individuellen Bildungschancen - eng mit der sozialstrukturellen Lage.

23 Der Hinweis auf Nutzungsrechte, analog zu den digitalen Zugriffsrechten, kann jedoch solange kaum überzeugen, solange die Angebote so uneinheitlich und so wenig nutzerfreundlich sind. Jeremy Rifkins access-These, mit der er eine generelle Ablösung des Eigentumsverhältnisses durch temporäre Verfügungsregime infolge der Digitalisierung und Tertiarisierung diagnostiziert (vgl. Rifkins 2000), ist gewagt. Die Eigentumsfrage im Verkehr stellt sich aber möglicherweise dann neu, wenn die Alternative schärfer konturiert ist: Möchte der hochaktive und eigenverantwortliche Urbanit in seiner auf Selbstbeweglichkeit und verkehrliche Autarkie gerichteten Grundhaltung lieber auf eine breite Palette von Verkehrsoptionen zugreifen können oder will er alle Verkehrsbedürfnisse mit seinem eigenen Auto abdecken, das notwendigerweise weniger Nutzungsvielfalt bietet? Ob das Pendel eher in Richtung eines weiteren Bedeutungszuwachses des privaten - und gegenüber den Zumutungen einer segmentierten Öffentlichkeit abschottenden - Autobesitzes oder in Richtung Nutzungsvielfalt durch neue intermodale Verkehrsdienstleistungen ausschlägt, dürfte nicht allein von angeblich anthropologischen Konstanten wie dem persönlichen Besitzdrang abhängen. Entscheidend könnte vielmehr sein, welche Angebote an integrierten Verkehrsdienstleistungen - inklusive stabilem Funktionsraum - tatsächlich zur Verfügung stehen werden und ob sie hinreichend zuverlässig, kostengünstig und routinefähig sind. 
Während auf der Mesoebene Hinweise für eine Heterogenisierung von formalen Regeln und Zugangsoptionen im (Auto)Verkehr und der wachsenden Bedeutung kognitiver Kompetenzen sowie auf eine Destandardisierung von Nutzungsoberflächen im Verkehr zu finden sind, ist global betrachtet auf der Makroebene zukünftiger (Auto)Mobilität eher von einer Angleichung auszugehen. Ganz im Sinne der neo-institutionalistischen Erwartungen der Verbreitung hegemonialer Konsum- und Produktbilder tritt das Auto als Symbol für ökonomischen Erfolg in den Ländern und Regionen der nachholenden Modernisierung auf. Trotz des forcierten Aufbaus einer eigenen Autoindustrie und trotz der teils staatlich geförderten Einführung eigener Modelle in China und Indien versprechen die etablierten Marken und Modelle aus den klassischen Wohlstandsregionen des Westens die höchsten Prestigegewinne. Ein Grund ist die anerkannte Qualität der Produkte, aber mindestens so relevant sind die Images und der Markenmythos westlicher Autofirmen. Insofern lässt sich die an westlichen Vorbildern orientierte Automobilisierung in den bisher kaum motorisierten Regionen der Welt als Teil einer umfassenden Globalisierung interpretieren. ${ }^{24}$

Auch die lange eingespielte stratifizierte Fahrzeug- und Modellabfolge - vom Fahrrad und Motorroller über das kleine Einstiegsmodell bzw. das Gebrauchtauto zum Mittelklasse- und später zum Oberklassefahrzeug - wird in den emerging countries weiterhin eingehalten. Größere Abweichungen oder gar ein eigener Mobilitätsweg sind bisher weder in den asiatischen Boomregionen noch in Südamerika oder etwa in Russland zu sehen. Der Blick der potenziellen Autokunden insgesamt und insbesondere der kaufkräftigen sozialen Aufsteiger ist auf die etablierten Autohersteller und ihre auf den westlichen Heimatmärkten erfolgreichen Modelle gerichtet.

\section{Zwischen Individualisierung und Vergemeinschaftung}

Moderne Gesellschaften unterscheiden sich von vormodernen Gesellschaften dadurch, dass sich ihre Mitglieder aus überkommenen Abhängigkeiten lösen und von starren sozialen Rollenzuweisungen befreien können. Tun sie das, werden sie mobil. ${ }^{25}$ Insbesondere ist der Automobilismus Produkt und Treiber der Moderne. Seine Ambivalenz ist mit Händen zu greifen. Sie ist ein anschauliches Beispiel für eine „duale Struktur“, der Giddens eine ermöglichende und zugleich eine determinierende Qualität beimisst. Während das Auto komplexe Alltagsabläufe für die und den Einzelnen erleichtert (oder erst ermöglicht) und Handlungsoptionen eröffnet, fördert es entfernungsintensive Lebens-, Produktions- und Distributionsformen und provoziert car dependency. Als verallgemeinerungsfähiges - und das kann nur heißen: als für die globale Umweltbalance verträgliches - Modell taugt es aufgrund seiner Externalitäten zudem nicht. Angestrebt wird es dennoch. Modernisierungsphasen, einhergehend mit der Bildung von Mittelschichten und wachsenden demokratischen Ansprüchen, sind bisher immer zugleich Phasen der automobilen Motorisierung.

24 Insofern kann das Automobil als Anschauungsmaterial für Krückens Charakterisierung des worldpolity-Ansatzes von John W. Meyer und seiner Schule dienen, in der er die diffundierende umfassende Rationalisierung (Weberscher Provenienz) als Grundströmung der Globalisierung ausweist: „Bei Meyer wird der prinzipiell unabgeschlossene Rationalisierungsproze $ß$ als Globalisierungsprozeß verstanden. In seinem Verlauf breiten sich grundlegende okzidentale Strukturmuster - die formale Organisation, das rationale Individuum - über den gesamten Erdball aus. Beschleunigt wird diese Diffusion dadurch, dass diese Muster mythisch überhöht, mit Symbolen unterlegt und ritualisiert in Szene gesetzt werden.“ (Krücken 2005: 301).

25 Stephan Rammler hat dieses Motiv in den Arbeiten der soziologischen Klassiker herausgearbeitet. Sowohl bei Weber als auch bei Simmel taucht es als implizites Element ihrer Modernisierungsanalysen auf, als unausgesprochenes Prinzip der Dynamisierungs- und Differenzierungsprozesse in sich modernisierenden Gesellschaften (Rammler 2001). 
Vor allem die Individualisierung bringt Möglichkeiten für eine höhere räumliche und soziale Beweglichkeit hervor und erzwingt diese teilweise auch. Die erhöhte Mobilität, verstanden als potenzielle - erwartete oder geforderte - Beweglichkeit, steht in einem wechselseitigen Verhältnis zum Verkehr. Denn die Bewegung in konkreten Räumen hängt von Wünschen, Sehnsüchten, Erwartungen und Kalkülen ab, die mental generiert werden. Die mentale Vorarbeit ihrerseits geschieht im Wissen um die technischen und organisatorischen Möglichkeiten gegebener Verkehrsangebote - und darüber hinaus gegebener informationstechnischer Anbindungen. Das Automobil bietet im Vergleich zu anderen Verkehrsmitteln viele Vorteile. Vor allem ist es flexibel einsetzbar und kann sowohl kurze als auch mittlere und lange Distanzen überwinden helfen.

Zugleich zeigt sich mit fortschreitender Individualisierung ein veränderter gesellschaftlicher Umgang mit Zeit und Raum. Ihre Wahrnehmung wird generell vielfältiger und eigensinniger (Knie 1997). Die Erosion klassischer Vergemeinschaftungsformen zeigt sich auch in der nachlassenden Disziplinierung durch kollektive Zeittakte. Eine ähnliche Entwicklung wie bei der Zeit kann auch für den Raum festgestellt werden. Der Bedarf an eigenkontrollierten Räumen wächst, der Raumkonsum steigt. Auch und gerade im Transferbereich, also im Verkehrsraum, besteht ein hohes Bedürfnis nach Privatheit in geschützten „Eigenräumen“.

Schließlich bedeutet Individualisierung auch die Ausweitung der Bandbreite persönlicher Aktivitätsoptionen - insbesondere in der Freizeit. Damit geraten erneut der Verkehr, seine Optionen, Verfügbarkeit, seine mentale Repräsentanz, nicht zuletzt seine Kosten und Kostenwahrnehmung, in den Fokus. Der schnelle Zugriff und unaufwendige Nutzungschancen von Verkehrsangeboten werden als Voraussetzung für Aktivitäten zur Selbstentfaltung betrachtet. Es gibt keine Hinweise darauf, dass ein erreichtes Niveau von bereits realisierter und biografisch ,erfahrener“ Mobilität freiwillig unterschritten wird.

Das Auto ist eine Technik, die zusätzliche individuelle Handlungsoptionen schafft und gesellschaftliche Teilhabe fördert, den Raumwiderstand senkt und damit sowohl sozialen Aufstieg erleichtert als auch eine komplexe Alltagsorganisation und die Trennung der Funktionsbereiche Arbeiten, Wohnen und Reproduktion ermöglicht. Die Automobilisierung ist Teil des westlichen Modells der Modernisierung, Ausdruck individuellen Aufstiegs ebenso wie Symbol für kollektive Fortschrittserwartungen. Das Auto ist, ob in den früh motorisierten Gesellschaften oder in denen inmitten einer nachholenden Modernisierung, nicht nur ein Hilfsmittel einer geglückten sozialen Integration, sondern auch ein Artefakt, das Handlungen prägt. Im Sinne des Giddenschen Strukturierungskonzeptes wird es oft - und vor allem in der alltäglichen Verwendung - routinemäßig benutzt. Zwanglos, aber regelmäßig unbewusst. Dadurch erhält es umgekehrt seine strukturierende Bedeutung für Alltagsabläufe, Residenzentscheidungen, Arbeitsplatzsuche, Freizeitpräferenzen etc.

Dennoch: widersprüchlicher kann der Befund kaum sein. Während die Grenzen des Wachstums beim Automobil infolge seines globalen Siegeszuges im dominanten Umwelt-, Ressourcen- und Klimadiskurs beinahe zum Allgemeingut geworden sind, schreitet die automobile Aufrüstung weiter voran. Und zwar nicht nur als nachholende Motorisierung in der aufstrebenden BRIC-Welt. Auf dem weithin gesättigten deutschen Markt beispielsweise ist die durchschnittliche Motorisierung der neu gekauften Pkw in gut eineinhalb Jahrzehnten von 1995 bis 2011 von 95 auf 134 PS gestiegen (vgl. FAZ 30.7.2011). Zweifel kommen angesichts dieser automobilen Hochrüstung auf, wie weit die ebenfalls beobachtbaren Verschiebungen in der symbolischen Bedeutung des Autos und die sich andeutenden Prozesse der Heterogenisierung und Differenzierung in Angebot und Nachfrage nach (Auto-)Mobilität tatsächlich reichen. Entweder bleiben sie auf überschaubare postmaterialistische urbane Milieus in den früh motorisierten Regionen der Welt beschränkt oder sie verbreiten sich und kündigen sogar weltweite Trends an. Zwar sind sowohl Konzepte von intermodalen Mobilitätsdienstleistungen 
als auch Elektroautos nicht vollkommen neu. Schon länger galten sie als potenzielle Geschäftsfelder für die reifen Autogesellschaften der Triade. Ein Ausbruch aus der Nische käme jedoch einem Pfadbruch im Automobilbau und einer Abkehr vom Prinzip des Universalautos mit dem Verbrennungsmotor als seinem technischen Kern gleich.

\section{Literatur}

Ahrends, Gerd-Axel (2009): Ergebnisse und Erkenntnisse zur Mobilität in Städten aus der Haushaltsbefragung SrV 2008, Präsentation auf der Abschlusskonferenz SrV 2008 am 30. Juni 2009 in Dresden.

Berger, Peter A. (1996): Individualisierung. Status, Unsicherheit und Erfahrungsvielfalt, Opladen.

Berger, Peter A. (2001): Soziale Mobilität, in: Bernhard Schäfers / Wolfgang Zapf (Hrsg.), Handwörterbuch zur Gesellschaft Deutschlands, Opladen, S. 595-605.

Berger, Peter L. / Thomas Luckmann (1969): Die gesellschaftliche Konstruktion der Wirklichkeit. Eine Theorie der Wissenssoziologie, Frankfurt / Main.

Bourdieu, Pierre (1979 / 1987): Die feinen Unterschiede. Kritik der gesellschaftlichen Urteilskraft, Frankfurt / Main.

Bourdieu, Pierre (1985): Sozialer Raum und ,Klassen'. Zwei Vorlesungen, Frankfurt / Main.

Bourdieu, Pierre (1998): Der Einzelne und das Eigenheim, Hamburg.

Burkart, Günter (1994): Individuelle Mobilität und soziale Integration. Zur Soziologie des Automobilismus, in: Soziale Welt 45, S. 216-241.

Canzler, Weert (2010): Mobilitätskonzepte der Zukunft und Elektromobilität, in: Reinhard Hüttl / Bernd Pischetsrieder / Dieter Spath (Hrsg.), Elektromobilität. Potenziale und wissenschaftlich-technische Herausforderungen, Heidelberg, S. 39-61.

Canzler, Weert / Frank Hunsicker / Astrid Karl / Andreas Knie / Ulrich König / Günter Lange / Christian Maertins / Lisa Ruhrort (2007): DB Mobility: Beschreibung und Positionierung eines multimodalen Verkehrsdienstleisters. InnoZ-Baustein Nr. 1, abrufbar unter: http://innoz.de/fileadmin/INNOZ/pdf/ Bausteine/innoz-baustein-01.pdf, letztes Abrufdatum: 1.8.2012.

Canzler, Weert / Andreas Knie (1998): Möglichkeitsräume. Grundrisse einer modernen Mobilitäts- und Verkehrspolitik, Wien - Köln - Weimar.

Canzler, Weert / Andreas Knie (2011): Einfach aufladen. Mit Elektromobilität in eine neue Zukunft, München.

Canzler, Weert / Lutz Marz (2011): Wert und Verwertung neuer Technologien. Das Beispiel der Wasserstoff- und Brennstoffzellentechnologien, in: Leviathan 39, S. 223-245.

Cass, Noel / Elisabeth Showe / John Urry (2005): Social Exclusion, Mobility and Access, in: Sociological Review 53, S. 539-55.

Claessens, Dieter (1966): Angst, Furcht und gesellschaftlicher Druck und andere Aufsätze, Dortmund.

Destatis (2012): Statisches Bundesamt: Preise. Harmonisierte Preisindizes. Dezember 2011, Wiesbaden, abrufbar unter: http: https://www.destatis.de/DE/Publikationen/Thematisch/Preise/Verbraucherpreise/ VerbraucherpreisindexJahresberichtPDF_5611104.pdf?_blob=publicationFile, letztes Abrufdatum: 1.1.2013.

DIW / Infas (2009 (Hrsg.): Mobilität in Deutschland 2008, Berlin - Bielefeld.

Eisenkopf, Alexander (2002): Effiziente Straßenbenutzungsabgaben. Theoretische Grundlagen und konzeptionelle Vorschläge für ein Infrastrukturabgabensystem, Giessener Studien zur Transportwirtschaft und Kommunikation (hrsg. von Gerd Aberle), Hamburg.

European Commission (2011): White paper. Roadmap to a Single European Transport Area - Towards a competitive and resource efficient transport system, COM (2011) 144, Brüssel.

Frankfurter Allgemeine Zeitung (FAZ) (30.7.2011): Mehr Pferdestärken denn je, S. 11. 
FES Friedrich-Ebert-Stiftung (2010): Zukunft der deutschen Automobilindustrie. Herausforderungen und Perspektiven für den Strukturwandel im Automobilsektor, Diskussionspapier der Arbeitskreise Innovative Verkehrspolitik und Nachhaltige Strukturpolitik der Friedrich-Ebert-Stiftung, Bonn.

Geels, Frank W. / René Kemp / Geoff Dudley / Glenn Lyons (Hrsg.) (2011): Automobility in Transition? A Socio-Technical Analysis of Sustainable Transport, London - New York / NY.

Geißler, Karlheinz A. (2004): Alles. Gleichzeitig. Und zwar sofort. Unsere Suche nach dem pausenlosen Glück, Freiburg.

Giddens, Anthony (1988 a): Die Konstitution der Gesellschaft, Frankfurt / Main - New York / NY.

Giddens (1988 b): Die „Theorie der Strukturierung“. Ein Interview mit Anthony Giddens, in: Zeitschrift für Soziologie 17, S. 286-295.

Gigerenzer, Gerd (2004): Fast and frugal heuristics: The tools of bounded rationality, in: Derek Koehler / Nigel Harvey (Hrsg.), Blackwell handbook of judgement and decision making, Oxford, S. 62-88.

Gottl-Ottlilienfeld, Friedrich von (1925): Fordismus, Jena.

Häußermann, Hartmut / Walter Siebel (1992): Soziologie des Wohnens, in: Hartmut Häußermann et al. (Hrsg.), Stadt und Raum. Soziologische Analysen, Pfaffenweiler, S. 69-116.

Harvey, David (1990): The Condition of Postmodernity. Cambridge - Oxford.

Helmers, Eckard (2009): Bitte wenden Sie jetzt. Das Auto der Zukunft, Weinheim.

Holzapfel, Helmut (1997): Autonomie statt Auto. Zum Verhältnis von Lebensstil, Umwelt und Ökonomie am Beispiel des Verkehrs, Bonn.

Institut für Mobilitätsforschung (Ifmo) (2011): Mobilität junger Menschen im Wandel, ifmo-Studien, München.

Joas, Hans (1992): Eine soziologische Transformation der Praxisphilosophie. Giddens' Theorie der Strukturierung, in: Ders., Pragmatismus und Gesellschaftstheorie, Frankfurt / Main, S. 205-222.

Kaufmann, Vincent (2002): Re-Thinking Mobility. Contemporary Sociology, Aldershot.

Kaufmann, Vincent / Gil Viry / Eric D. Widmer (2010): Motility, in: Norbert F. Schneider / Beate Collet (Hrsg.), Mobile Living Across Europe II. Causes and Consequences of Job-Related Spatial Mobility in Cross-National Camparison, Opladen - Farmington Hills / MI, S. 95-111.

Kesselring, Sven (2009): Global Transfer Points: The Making of Airports in the Mobile Risk Society, in: Saulo Cwerner / Sven Kesselring / John Urry (Hrsg.), Aeromobilities, London - New York / NY.

Kesselring, Sven (2012): Betriebliche Mobilitätsregime. Zur sozio-geografischen Strukturierung mobiler Arbeit, in: Zeitschrift für Soziologie 41, S. 83-100.

Kesselring, Sven / Gerlinde Vogl (2010): Betriebliche Mobilitätsregime, Berlin.

Knie, Andreas (1997): Eigenzeit und Eigenraum, in: Soziale Welt 47, S. 39-55.

Knie, Andreas / Otto Berthold / Sylvia Harms / Bernhard Truffer (1999): Die Neuerfindung urbaner Mobilität. Elektroautos und ihr Gebrauch in den USA und Europa, Berlin.

Knöbl, Wolfgang (2007): Die Kontingenz der Moderne. Wege in Europa, Asien und Amerika, Frankfurt / Main.

Knoflacher, Hermann (2009): Virus Auto. Die Geschichte einer Zerstörung, Wien - Köln - Weimar.

Kraftfahrtbundesamt (KBA) 2010: Jahresbericht 2009, abrufbar unter: http://www.kba.de/cln_015/ nn_124384/DE/Presse/Jahresberichte/jahresbericht_2009_ pdf,templateId=raw, property=publicationFile.pdf/jahresbericht_2009_pdf.pdf, letztes Abrufdatum: 1.1.2012.

Krücken, Georg (2005 a): Einleitung in den Band, in: John W. Meyer, Weltkultur. Wie die westlichen Prinzipien die Welt durchdringen, (hrsg. von Georg Krücken), Frankfurt / Main, S. 7-16.

Krücken, Georg (2005 b): Der ,,world-polity“-Ansatz in der Globalisierungsdiskussion, in: John W. Meyer, Weltkultur. Wie die westlichen Prinzipien die Welt durchdringen, (hrsg. von Georg Krücken), Frankfurt / Main, S. 299-318. 
Krücken, Georg / Raimund Haase (2005): Neo-Institutionalismus, Bielefeld.

Kutter, Eckhard (1975): Mobilität als Determinante städtischer Lebensqualität, in: Wilhelm Leutzbach (Hrsg.), Verkehr in Ballungsräumen, Schriftenreihe der DVWG, Bd. 24, Köln, Berlin, S. 65-75.

Kutter, Eckhard (2007): Raum und Verkehr, in: Oliver Schöller / Weert Canzler / Andreas Knie (Hrsg.), Handbuch Verkehrspolitik, Wiesbaden, S. 252-278.

Larsen, Jonas / John Urry / Kai W. Axhausen (2006): Mobilities, Networks, Geographies, Aldershot.

Latour, Bruno (1996): On Actor Network Theory. A Few Clarifications, in: Soziale Welt 47, S. 369-381.

Latour, Bruno (2005): Reassembling the Social. An Introduction to Actor-Network-Theory, Oxford.

Löw, Martina (2001): Raumsoziologie, Frankfurt / Main.

Lutz, Burkard (1984): Der kurze Traum immerwährender Prosperität, Frankfurt / Main.

Mau, Steffen (2007): Transnationale Vergesellschaftung. Die Entgrenzung sozialer Lebenswelten, Frankfurt / Main.

Matthies, Hilde (2009): Öffentliche Leihfahrräder in Europa. Ein Überblick, Präsentation am 7.8.2009 auf dem Nationalen Fahrradcongress in Berlin, abrufbar unter: http://www.google.de/search?q=matthies +hildegard+2009+bike\&sourceid=ie7\&rls=com.microsoft:en-US\&ie=utf8\&oe=utf\&\&redir_esc $=\&$ ei $=$ cgt6Tp2vJs_Zsgb5srSRDw, letztes Abrufdatum: 1.1.2012.

Meyer, John W. (2005): Weltkultur. Wie die westlichen Prinzipien die Welt durchdringen, (hrsg. von Georg Krücken), Frankfurt / Main.

Meyer, John W. / John Bohli / George M. Thomas (2005): Ontologie und Rationalisierung im Zurechnungssystem der westlichen Kultur, in: John W. Meyer, Weltkultur. Wie die westlichen Prinzipien die Welt durchdringen, (hrsg. von Georg Krücken), Frankfurt / Main, S. 17-46.

Nieuwenhuis, Paul / Peter Wells (2008): The automotive industry and the environment. A technical, business and social future, Cambridge.

Ohnmacht, Timo / Andreas Frei / Kai W. Axhausen (2008): Mobilitätsbiografie und Netzwerkgeografie: Wessen soziale Beziehungen sind räumlich dispers?, in: Schweizerische Zeitschrift für Soziologie 34, S. 131-164.

Rammler, Stephan (2001): Mobilität in der Moderne. Geschichte und Theorie der Verkehrssoziologie, Berlin.

Reheis, Fritz (1998): Die Kreativität der Langsamkeit. Neuer Wohlstand durch Entschleunigung, Darmstadt.

Rifkin, Jeremy (2000): The Age of Access, New York / NY.

Rust, Holger (2011): Das kleine Schwarze. Jugendliche Autoträume als Herausforderung für das Zukunftsmanagement, Wiesbaden.

Sachs, Wolfgang (1984): Die Liebe zum Automobil. Ein Rückblick in die Geschichte unserer Wünsche, Reinbek.

Schade, Wolfgang / Anja Peters / Claus Doll / Stefan Klug / Jonathan Köhler / Michael Krail (2011): VIVER - Vision für nachhaltigen Verkehr in Deutschland, Working Paper Sustainability and Innovation S 3 / 2011, Karlsruhe.

Schroer, Markus (2006): Räume, Orte, Grenzen. Auf dem Weg zu einer Soziologie des Raums, Frankfurt / Main.

Schulze, Gerhard (1992): Die Erlebnisgesellschaft. Kultursoziologie der Gegenwart, Frankfurt / Main.

Sennett, Richard (1998): Der flexible Mensch. Die Kultur des neuen Kapitalismus, Berlin.

Sheller, Mimi (2008): Gendered Mobilities: Epilog, in: Tanu Prya Uteng / Tim Cresswell (Hrsg.), Genderd Mobilities, Aldershot, S. 257-265.

Sieverts, Thomas (1998): Zwischenstadt zwischen Ort und Welt, Raum und Zeit, Stadt und Land, Braunschweig. 
Solga, Heike / Justin Powell / Peter A. Berger (Hrsg.) (2009): Soziale Ungleichheit. Klassische Texte zur Sozialstrukturanalyse, Franfurt / Main - New York / NY.

Tully, Claus (2011): Mobilisierung des Mobilen. Trends in der Jugendmobilität, in: Der Nahverkehr 29, S. 12-15.

Tully, Claus (2012): Jugend und Mobilität. in: Rauschenbach, Thomas et al. (Hrsg.), Handbuch Jugend Evangelische Perspektiven, München.

Tully, Claus / Claudio Alfaraz (2010): Technikbasierte Raumbezüge im Jugendalltag, in: deutsche jugend 58, S. 122-128.

Ullrich, Otto (1988): Industrielle Lebensweise und das Automobil, in: Helmut Holzapfel (Hrsg.), Ökologische Verkehrsplanung, Menschliche Mobilität, Straßenverkehr und Lebensqualität, Frankfurt / Main.

Urry, John (2004): The ,System' of Automobility, in: Theory, Culture and Society 21, S. 25-39.

Urry, John (2007): Mobilities, Cambridge.

Urry, John (2012): Does Mobility Have a Future?, in: Margaret Grieco / John Urry (Hrsg.), Mobilities: New Perspectives on Transport and Society, Aldershot, S. 3-19.

Voy, Klaus / Werner Polster / Claus Thomasberger (Hrsg.) (1991): Gesellschaftliche Transformation und materielle Lebensweise, Marburg.

Zumkeller, Dirk / Wilko Manz / Jörg Last / Bastian Chlond (2005): Die intermodale Vernetzung von Personenverkehrsmitteln unter Berücksichtigung der Nutzerbedürfnisse (INVERMO), Schlussbericht, Karlsruhe, abrufbar unter: http://verkehrspanel.ifv.uni-karlsruhe.de/informationen/indexinformationen.htm, letztes Abrufdatum: 5.1.2013.

Dr. Weert Canzler

Wissenschaftszentrum Berlin für Sozialforschung Forschungsgruppe Wissenschaftspolitik

Reichpietschufer 50

10785 Berlin

weert.canzler@wzb.eu 